\title{
Effect of age and other factors on macular pigment optical density measured with resonance Raman spectroscopy
}

\author{
Akira Obana • Yuko Gohto • Masaki Tanito • \\ Shigetoshi Okazaki • Werner Gellermann • \\ Paul S Bernstein • Akihiro Ohira
}

Received: 3 September 2014 / Accepted: 5 September 2014 /Published online: 9 October 2014

(C) Springer-Verlag Berlin Heidelberg 2014

We appreciate the letter from Kawada and are pleased to learn that he is interested in our manuscript.

As he pointed, the coefficient of determination of multiple regression analysis is not high $\left(R^{2}=0.1391\right)$. We discussed this issue in the discussion on page 1226 and 1227 . The explanatory variables that were not included in the present analysis would be a reason for the small correlation coefficient. The amount of intake of lutein and zeaxanthin and subject's genetic background are thought to be important variables. The details are described in the discussion, and the BMI was treated numerically.

The issue of lutein in the brain and cognitive function is a new and very important topic. We agree with Kawada and we also hope new knowledge on this issue will be obtained in the near future.
The relationship between aging and macular pigment optical density (MPOD) levels has been addressed in many studies using different methods to measure MPOD levels in a limited number of subjects. Our previous study using resonance Raman spectroscopy (RRS) in 100 healthy subjects revealed age-related decline in MPOD levels, but there was a possibility that age-related yellowing of the human lens would affect the results. Then we performed the present study on eyes with clear intraocular lenses. As described in the final paragraph, we believe that MPOD levels measured by RRS decrease with aging in the range of ages that we investigated. The purpose and significance of the present study was described in its introduction.
A. Obana $(\bowtie) \cdot$ Y. Gohto

Department of Ophthalmology, Seirei Hamamatsu General Hospital, Sumiyoshi, 2-12-12, Naka-ku, Hamamatus City, Sizuoka 430-8558, Japan

e-mail: obana@sis.seirei.or.jp

\section{A. Obana $\cdot$ S. Okazaki}

Department of Medical Spectroscopy, Applied Medical Photonics Laboratory, Medical Photonics Research Center, Hamamatsu University School of Medicine, Handayama1-20-1, Higashi-ku, Hamamatsu City, Shizuoka 431-3192, Japan

M. Tanito $\cdot$ A. Ohira

Department of Ophthalmology, Shimane University Faculty of

Medicine, Shiojimachi 89-1, Izumo City, Shimane 693-8501, Japan

W. Gellermann

Department of Physics and Astronomy, University of Utah, 115S

1400 E RM 201, Salt Lake City, UT 84112, USA

P. S. Bernstein

Moran Eye Center, University of Utah School of Medicine, 65 Mario

Capecchi Drive, Salt Lake City, UT 84132, USA 